\title{
Bound states of electrons with soliton-like excitations in thermal systems - adiabatic approximations *
}

\author{
W. Ebeling ${ }^{1}$, M.G. Velarde ${ }^{2}$, A.P. Chetverikov ${ }^{3}$ \\ ${ }^{1}$ Institute of Physics, Humboldt University, Berlin \\ 2 Instituto Pluridisciplinar, Universidad Complutense, Madrid \\ 3 Institute of Physics, Saratov State University, Saratov
}

Received June 23, 2009

\begin{abstract}
We study the bound states of electrons with solitonic excitations in one and two-dimensional atomic systems. We include Morse interactions between the atoms in a temperature range from low to physiological temperatures. The atoms are treated by classical Langevin equations. In a first approach, the places of compressions are visualized by drawing the overlap of the densities of the core electrons. Then we study the effect of nonlinear vibrations on the added, free electrons moving on the background of the atoms. We study the coupled classical and quantum-mechanical dynamics of the electrons on the lattice in tight-binding approximation (TBA). Further we consider the formation of solectrons, i. e., dynamic electron-soliton bound states in adiabatic approximations based on the energy spectrum and the canonical distribution.
\end{abstract}

Key words: nonlinear lattice, electron lattice interactions, solectrons, adiabatic approximations

PACS: 63.20.Ry, 05.60.-k, 05.45.Yv, 71.38.-k

\section{Introduction}

Already the first work of Bogolyubov on statistical physics which appeared in 1946 was fundamental for the development of our science [1]. When in 1958 M.M. Bogolyubov came to Berlin on the occasion of Planck's 100th birthday, he left behind a copy of his book. Fortunately, one of the present authors (W.E.) came across this copy and was fascinated by Bogolyubov's method and carried out a diploma research on corresponding applications. In the academic year of 1960/61 he was lucky to hear the lectures by Bogolyubov at Moscow State University and went into his lecture notes on quantum statistics [1]. By chance in the same academic year he could also listen to the lectures by another great pioneer of quantum statistics A.S. Davydov.

We mention this here because the present work is closely related to the work performed by these great pioneers, and, in particular, to the models developed by Davydov [2]. In his seminal work Davydov studied the (nonlinear) interaction between (linear) lattice vibrations and electronic states and transitions in a lattice. Since the publication of this work by Davydov and others, it is known that due to the nonlinearity induced by the electron-(acoustic) phonon interaction (polaron or the so-called electron self-trapping effect), the excitations which he called "electro-solitons" may travel along the dynamically harmonic lattices. Davydov argued that these excitations could be stable at finite temperatures and could persist even at physiological temperatures. Several authors have checked this conjecture and have found that Davydov's electro-solitons are destroyed already around $10 \mathrm{~K}$ lasting at most $2 \mathrm{ps}[3-5]$. Here we shall address Davydov's conjecture but rather than using a harmonic lattice we shall consider anharmonic lattice dynamics. It is now well established that if the underlying lattice dynamics involves anharmonic interaction this may result in the appearance of very stable supersonic acoustic solitons running free along the lattice like in a Toda lattice [6-10]. The situation in two-dimensional or three-dimensional systems with hard repulsive interactions is less clear. However, there are several theoretical and simulation

*Dedicated to the 100th birthday of M.M. Bogolyubov, submitted to the Proceedings of StatPhys'2009. 
results which show that soliton-like excitations occur [11,12]. For example for a special nonlinear lattice, a continuous description of the nonlinear excitations is possible by some generalization of the Kadomtsev-Petiashvili equation which possesses soliton-like solutions [13]. This and other papers, e. g., $[14,15]$ give us a hint that indeed in two-dimensional systems soliton-like excitations occur. Some evidence for the occurrence of thermal compression waves also comes from computer simulations for two-dimensional Lennard-Jones systems [16-18]. In these systems energy-rich hard excitations were observed which are capable of activating the imbedded impurities.

Let us now discuss the coupling of soliton-like excitations to the imbedded electrons. After Davydov's pioneering work many investigations were performed for one-dimensional lattices with Morse interactions and imbedded electrons [19-22]. Here we shall follow this idea studying the systems with Morse interactions together with an appropriate electron-atom interaction. These potentials can be scaled around the minimum in such a way that the first three derivatives are identical to Lennard-Jones systems which guarantees a close relationship of their nonlinear (soliton) excitations [9]. It is also known that these excitations bring in a new form of dressed electrons or electro-soliton dynamic bound states [23-25]. They were called "solectrons" to mark the difference with Davydov's original electro-solitons. Then we shall show that due to the anharmonicity and the excitation of solitons, the thermal stability is considerably enhanced, in fact up to the room temperature range for biomolecules, for example.

\section{Langevin dynamics of the atoms}

\subsection{Hamiltonian and interaction potential}

In a mixed classical-quantum description we shall consider a $1 \mathrm{~d}$ or $2 \mathrm{~d}$ molecular system with the added (free) conduction electrons allowing the electron transfer (ET) or electric current in the presence of an external field. A wealth of information on both items in biomolecules and a diversity of approaches taken can be found in the references [28-34]. The system consists of $N$ classical units (atoms or molecules) and one or more non-interacting free electrons. We shall focus on the case of periodic boundary conditions. The electrons are allowed to occupy some $3 \mathrm{~d}$ volume surrounding the $1 \mathrm{~d}$ or $2 \mathrm{~d}$ system to form Coulomb fields. For the heavier lattice units (relative to the electrons) we shall assume equal masses $m$, coordinates $\mathbf{x}_{n}(t)$, velocities $\mathbf{v}_{n}(t), n=1, \ldots, N$ and a Hamiltonian

$$
H_{a}=\frac{m}{2} \sum_{n} \mathbf{v}_{n}^{2}+\frac{1}{2} \sum_{n, j} V\left(\mathbf{x}_{n}, \mathbf{x}_{j}\right)
$$

The subscripts locate atomic sites and the corresponding summations run from 1 to $N$. In the one-dimensional case, the mean equilibrium distance (lattice constant) between the particles in the lattice is denoted by $\sigma=L / N)$. In higher dimension we define the mean distance $\sigma$ by $V=$ $N(4 \pi / 3) \sigma^{3}$. We shall assume that the atomic particles repel each other by a strong (exponential) repulsive force and attract each other by a weak dispersion force with a potential which depends on the relative distance $r=\left|\mathbf{x}_{n+1}-\mathbf{x}_{n}\right|$ between the neighbors. As indicated earlier, we shall take the Morse function, probably the earliest quantum-mechanics based interaction potential

$$
V(r)=D\{\exp [2 B(r-\sigma)]-2 \exp [-B(r-\sigma)]\} .
$$

Exponentials are easily implemented in computers and they are also easy to handle mathematically. For illustration in our computer simulations we shall use $N=200$ and $B=1 / \sigma$. $B$ accounts for the stiffness of the interactions and $D$ provides an estimate of the binding/break-up energy of the bonds. Then, in the presence of random forces (hence non-zero temperature), the evolution of lattice particles is described by the (Newton-)Langevin equations $(n=1,2, \ldots, N)$

$$
\frac{\mathrm{d} \mathbf{v}_{n}}{\mathrm{~d} t}+\frac{1}{m} \frac{\partial H}{\partial \mathbf{x}_{n}}=-\gamma_{0} \mathbf{v}_{n}+\sqrt{2 D_{\mathrm{v}}} \xi_{n}(t) .
$$

We assume that the stochastic force $\sqrt{2 D_{\mathrm{v}}} \xi_{n}(t)$ models a surrounding heat bath (Gaussian white noise). The parameter $\gamma_{0}$ describes the common standard friction frequency acting on the lattice 
units or atoms from the surrounding heat bath. The validity of an Einstein relation is assumed $D_{\mathrm{v}}=$ $k_{\mathrm{B}} T \gamma_{0} / m$, where $T$ is temperature. In most cases we shall use $1 / B$ to denote the length unit (though we may occasionally use $1 / \sigma$ ) and the frequency of oscillations around the potential minimum $\omega_{0}^{-1}$ is the time unit. In the one-dimensional case typical parameter values for biomolecules are $\sigma \simeq 1-5 \AA ; B \simeq 1-5 \AA^{-1} ; D \simeq 0.1-0.5 \mathrm{eV}[31-34]$. This means that $B \sigma \simeq 1-25$ and $1 / \omega_{0} \simeq 0.1-0.5 \mathrm{ps}$. As the energy unit we shall use $2 D=m \omega_{0}^{2} \sigma^{2} /(B \sigma)^{2}$, that with $B \sigma=1$ reduces to $m \omega_{0}^{2} \sigma^{2}$, commonly used by most authors. This energy will be also used as a unit to measure the temperature $T\left(k_{\mathrm{B}}=8.6 \cdot 10^{-5} \mathrm{eV} / \mathrm{K} ; k_{\mathrm{B}} T=2 D\right)$.

\subsection{Solitonic excitations in one-dimensional systems and visualization}

In the earlier work we studied the connection between specific heat (at constant volume/length) of a system (1)-(3) and solitonic excitations [10]. Evidently, the nonlinear region lies where the thermodynamic quantities such as the mean potential energy per particle and the specific heat deviate from the corresponding values for an ideal lattice $u_{\text {pot }}=k_{\mathrm{B}} T / 2$ and $C_{\mathrm{v}}=k_{\mathrm{B}}$. The potential energy per particle is shown in figure 1 for demonstration. Since the deviations from the values for an ideal lattice is due to nonlinear effects, we expect that the number of solitons will increase in that region. This way, the number of solitons depends on the temperature and the nonlinear effects increase with the deviations of the thermodynamic functions from the values for an ideal lattice. In an infinite lattice with Toda interactions (and the like for a Morse potential) a soliton is described by

$$
\exp \left[-b\left(q_{n}-q_{n-1}\right)\right]=1+\beta_{0}^{2} \cosh ^{-2}\left[\kappa n-\beta_{0} t\right] .
$$

The total number of solitons can be approximated by $[7,8]$

$$
n(T) \simeq \operatorname{const} T^{1 / 3} \text {. }
$$

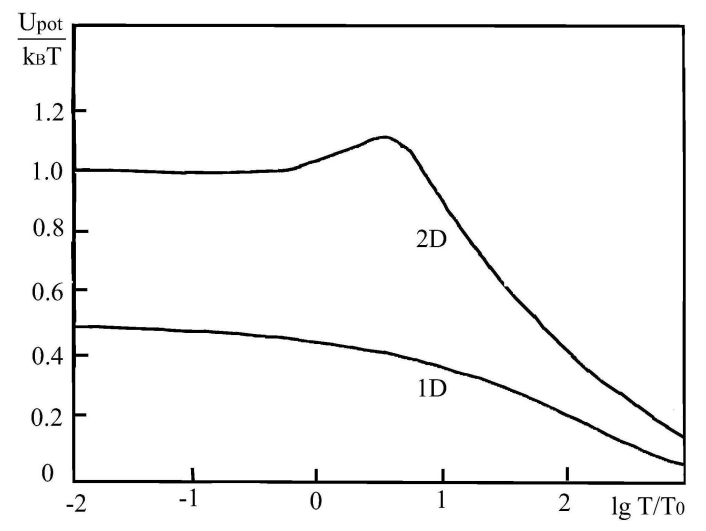

Figure 1. The mean potential energy per particle as a function of the temperature (in logarithmic scale) estimated from simulations for a one-dimensional Morse lattice [10] and for a two-dimensional fluid [17].

We may also estimate the frequency of solitons with a given width parameter $\kappa$ from the thermal statistics of the solitons in the lattice as done in $[7,8]$ for Toda interactions. If single solitons with parameter $\kappa$ are described by equation (27) then the density of solitons depending on parameter $\kappa$ reads according to $[8]$ :

$$
n(\kappa, T)=\frac{4 a \kappa}{\pi k_{\mathrm{B}} T} \exp (-\kappa) \exp \left[-(E(\kappa)-2 \kappa) / k_{\mathrm{B}} T\right]
$$


where

$$
E(\kappa)=\frac{2 a}{b}[\sinh \cosh \kappa-\kappa]
$$

According to these estimates, the number of solitons appears to increase with increasing temperature. On the other hand, their contribution to macroscopic properties, e. g., the specific heat, goes down. Therefore, we expect that there exists a kind of "optimal temperature" where solitons have the strongest effect $[7,8,22]$.

Accordingly, the region where anharmonicity plays a significant role is $0.35<u_{\text {pot }}<0.45$ or correspondingly $0.75<C_{\mathrm{v}} / k_{\mathrm{B}}<0.95$ (see figure 1 ). With increasing temperature, the effects of nonlinearities start with the multiphonon range or highly deformed-phonons domain on the way to melting in the system (recall that at high $T, u_{\text {pot }}=0$ and $c_{\mathrm{v}}=0.5$, there is a transition to a hard-sphere gas-like fluid phase). The corresponding temperatures in our energy units are in the range $T \simeq 0.1-0.5$ (and even up to $1-2$ ). Introducing the binding strength of the Morse lattice, as the Morse potential can be suitably adapted to the Toda interaction [22], we expect that solitonic effects should be in the range $T_{\text {sol }}^{\mathrm{M}} \simeq 0.2-1.0 D$. In electron volts $(\mathrm{eV})$ this would be the range $T_{\mathrm{sol}}^{\mathrm{M}} \simeq 0.01-0.1 \mathrm{eV}$. This range of temperatures for biomolecules includes the range of physiological temperatures (about $300 \mathrm{~K}$ ).

We can visualize the time evolution of the lattice atoms and hence the lattice deformations or lattice excitations by representing the density of the valence electrons which are moving bound to the ion cores. This can be achieved by considering, for simplicity, that each lattice unit is surrounded by a Gaussian electron density (atomic density) of width $s=0.35 \sigma$. Then, the total atomic electron density is given by

$$
\rho(\mathbf{x})=\sum_{n} \frac{1}{\sqrt{2 \pi} s} \exp \left[-\frac{\left(\mathbf{x}-\mathbf{x}_{n}(t)\right)^{2}}{2 s^{2}}\right] .
$$

Thus, each lattice atom is like a spherical unit with continuous (valence) electron density concentrated around its center. In regions where the atoms overlap, the density is enhanced. This permits to identify solitonic excitations based on a color code in a density plot. This is, of course, a rough approximation which helps visualization of the location of dynamic excitations by using the (covalence) electrons density enhancement as an alternative to mechanical lattice compression. This suffices for our purposes in section 2. The quantum-mechanical approach is used in sections 3 and 4. In figure 2 we show the result of computer simulations of a 1d Morse lattice with 200 particles for two temperatures $T=0.005(\sim 10 \mathrm{~K})$, and $T=0.1\left(\sim 2 \cdot 10^{2} \mathrm{~K}\right)$. The given absolute temperatures correspond to $D=0.1 \mathrm{eV}$. The diagonal stripes at $T=0.1$ correspond to the regions of enhanced
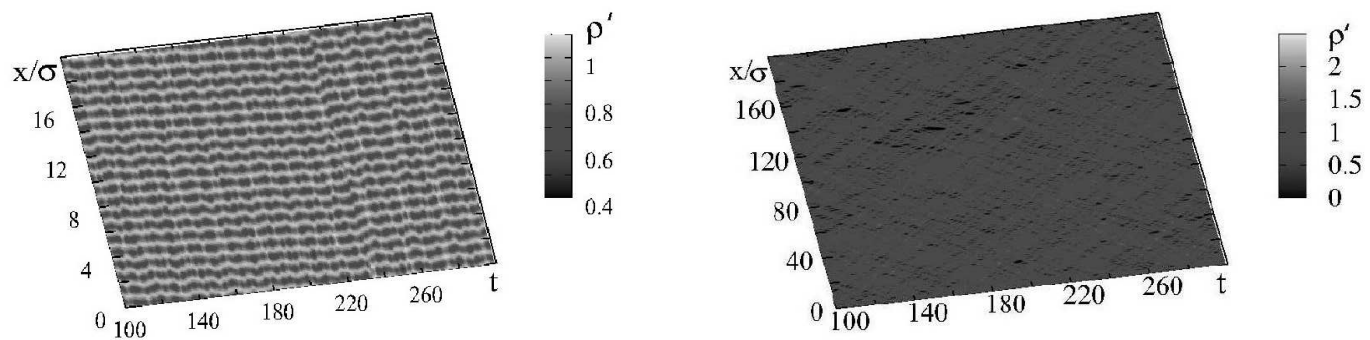

Figure 2. One-dimensional Morse lattice, positions over time. Visualization of running excitations (phonons and solitons) along the lattice through the density of the core electrons. The left panel corresponds to a rather low temperature $T=0.005$ (given in units of $2 D$ ): we see only harmonic lattice vibrations or phonons and no evidence of strong (soliton-like) excitations. At a higher temperature $T=0.1$ : several density peaks correspond to solitons (diagonal stripes). The strongest compressions move with velocity around $1.1 v_{\text {sound }}$. Parameter values: $N=200$ and $B \sigma=1$. 
density which are freely running along the lattice. This is the sign of solitonic excitations. Checking the slope we see that the excitations which survive more than 10 time units move with supersonic velocity. The pictures shown are quite similar to those described by Lomdahl and Kerr $[4,5]$ who gave a life-time of at most $2 \mathrm{ps}$ and which are stable only up to $10 \mathrm{~K}$. Ours, however, live about 10-50 time units, that is, for several picoseconds. Besides, they survive even at $T=1$ which is well above physiological temperatures. This confirms an earlier finding were at $T \simeq 300 \mathrm{~K}$ stable solitons and solectrons (dynamic electron-lattice soliton bound states) could be identified [24].

\subsection{Nonlinear excitations in two-dimensional systems}

In two-dimensional systems, nonlinear effects also play an essential role. We demonstrated this in figure 1 on the example of the specific heat, taken from an earlier work based on molecular dynamics simulations for Lennard-Jones-like molecules on a plane [16-18]. The calculation of the mean potential energy per molecule shows deviations from the ideal low-temperature Dulong-Petit value for a linear lattice, first an increase with temperature and only then a decrease to the ideal gas value. This clearly shows the occurrence of nonlinear effects. However, the situation in two-dimensional systems is quite different from one-dimensional lattice since the hard excitations in 2d-systems are quite different. However, we may still find compression waves running with approximately the sound velocity. As shown by Astakhova and Vinogradov [13] for a special nonlinear lattice, a continuous description of the nonlinear excitations is possible by some generalization of the Kadomtsev-Petiashvili equation which possesses soliton-like solutions. This and other papers give us a hint, that indeed in two-dimensional systems soliton-like excitations occur. In our context it is irrelevant whether these excitations are cnoidal waves in the mathematical sense or not. What is really relevant in this context is only whether these excitations are connected with local compressions which are running through the system with sound velocity or even with supersonic speed. Further, it is of relevance whether these excitations are observed in thermal systems. Some evidence for the occurrence of such thermal compression waves comes from earlier simulations for two-dimensional Lennard-Jones systems [16-18]
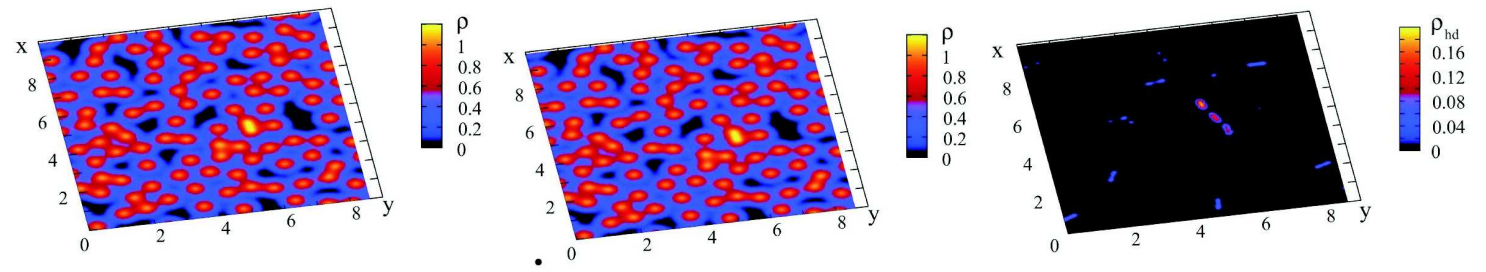

Figure 3. Two-dimensional system of 100 Morse molecules $(B \sigma=2)$ with periodic boundary conditions heated to the temperature $T=0.2$ (in units of $2 D$ ). Visualization of excitations (phonons and solitons) leading to a local increase of the density of the core electrons. We show in the left and center panels the evolution of the density distribution at two subsequent times and see the moving density waves. In the right panel, we demonstrate the time evolution of the highest peaks of density by showing density peaks at three subsequent times in one figure. One sees that the strongest compressions are soliton-like. These compression waves move with velocity around $1.2 v_{\text {sound }}$ and have a lifetime of a few time units.

We visualized the excitations in a two-dimensional system of Morse molecules in figure 3 by representing the increase of density of the core electrons. The first two figures (above) show the evolution of the density distribution by snapshots at 2 subsequent times (distance 1 time unit). In order to see the running density waves we showed in the panel below the time evolution of the highest peaks of density. From the shift of the peaks and the time distance between the snapshots we may estimate the velocity and find that the strongest compressions are soliton-like. They move with velocity around $1.2 v_{\text {sound }}$ and have a lifetime of a few time units. Similar to the $1 \mathrm{~d}$-case, the quasi-linear stripes in the snapshots correspond to the regions of enhanced density which are 
running through the system, which we consider to be a sign of soliton-like excitations.

\section{Electron dynamics and interaction with the atoms}

\subsection{Interaction by polarization effects and Boltzmann approximation}

Let us now introduce a free electron into the system. Among the variety of possible interactions here we consider only polarization effects. Our physical picture is based on the idea that the electrons near an atom give rise to a polarization energy. Let us assume that the electron is located at the position $\mathbf{x}$ and several atoms are located at nearby positions $\mathbf{x}_{j}$. For the polarization energy of the electron we may use the standard "ansatz"

$$
U(\mathbf{x})=U_{0}-U_{\mathrm{e}} \sum_{j \neq n}^{\prime} \frac{r_{0}^{4}}{\left[\left(\mathbf{x}-\mathbf{x}_{j}\right)^{2}+r_{0}^{2}\right]^{2}}
$$

Here $r_{0}$ is a characteristic distance and $U_{\mathrm{e}}$ is the maximal polarization energy. In the earlier work we also used a different pseudopotential approach $[22,26]$. In order to be consistent with the pseudopotential formula earlier used up to quadratic terms we can make the choice $r_{0}=2 h$.

Looking at the formula (9) we see that any cluster of atoms generates a potential hole in which electron density might be concentrated. Further any displacement of the atoms changes the polarization energy. The electron will try to follow up these changes. This is the basic effect leading to the solectron formation. In the simplest classical approximation we may assume that the electron density follows the Boltzmann distribution

$$
n(\mathbf{x})=\frac{\exp (-\beta U(\mathbf{x}))}{\int \mathrm{d} \mathbf{x}^{\prime} \exp \left(-\beta U\left(\mathbf{x}^{\prime}\right)\right)} .
$$

An example of a Boltzmann distribution of the electron density is demonstrated in figure 4.

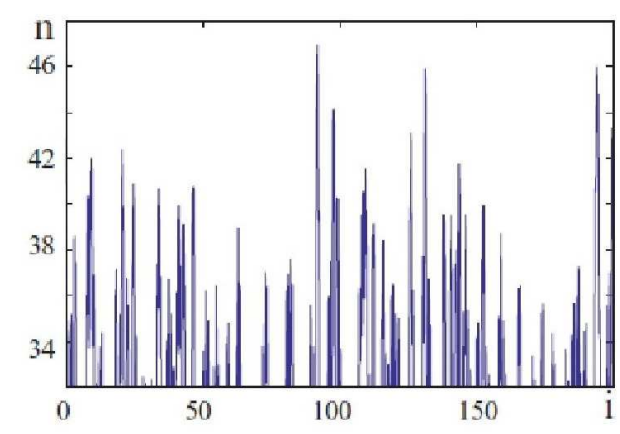

Figure 4. Boltzmann distribution of electrons in a 1d-system of 200 Morse atoms which are polarized by the electrons $T=0.1$.

\subsection{One-dimensional electron dynamics in tight-binding approximation}

Let us now go deeper into the question of solectron formation by placing the electrons on the lattice using the tight-binding approximation. The tight-binding approximation (TBA) replaces the Schrödinger continuum dynamics by a hopping process along the discrete lattice sites. Assuming that there is only one atomic state per lattice unit we get for the electrons the following Hamiltonian

$$
H_{\mathrm{el}}=\sum_{n}\left[E_{n}\left(\ldots, x_{n-1}, x_{n}, x_{n+1}, \ldots\right) c_{n}^{+} c_{n}-V_{n, n-1}\left(x_{n}, x_{n-1}\right)\left(c_{n}^{+} c_{n-1}+c_{n} c_{n-1}^{+}\right)\right] .
$$


Recall that (11) refers to initially free or excess electrons added to the lattice with atoms assumed to be located at sites $n$. The quantities $c_{n}, c_{n}^{+}$originally refer to Fermion destruction and creation operators, respectively, with appropriate anticommutation relations but here they are just complex numbers. Assuming that the interaction depends exponentially on the distance between the lattice units, we set

Then, the Hamiltonian (11) becomes

$$
V_{n, n-1}=-V_{0} \exp \left[-\alpha\left(q_{n}-q_{n-1}\right)\right]
$$

$$
H_{\mathrm{el}}=\sum_{n}\left\{\left(E_{n}^{0}+\delta E_{n}\right) c_{n}^{+} c_{n}-V_{0} \exp \left[-\alpha\left(q_{n}-q_{n-1}\right)\right]\left(c_{n}^{+} c_{n-1}+c_{n} c_{n-1}^{+}\right)\right\},
$$

where, for convenience in notation, $q_{n}$ denotes a lattice site spatial vibration (relative displacement) coordinate defined by $x_{n}=n \sigma+q_{n} / B$. The term $E_{n}^{0}=E_{0}$ denotes on-site energy levels of the unperturbed lattice which is independent of the site. Further $\delta E_{n}$ is the perturbation due to the lattice vibrations (harmonic as well as anharmonic modes may contribute). The simplest "ansatz" is linear in the deformations $\delta E_{n}=\chi_{0}\left(q_{n} / B\right)$, where the "electron-phonon coupling constant", $\chi_{0}$, indicates that the on-site energy level $E_{n}$, i. e., the local site energy, depends on the displacement of the unit at that site; $q_{n}$ is dimensionless (unit: $1 / B$ ). As shown e. g. in $[33,34]$, this coupling between lattice deformations and electronic states, for large enough values of the parameter $\chi$ leads to the formation of polarons. In view of the above given parameter values, the value of the coupling constant is in the range $\chi \simeq 0.1-2 \mathrm{eV} / \AA$. We have to take into account that our model is translationally invariant and we are considering relative lattice displacements. Accordingly, we set

$$
\delta E_{n} \simeq \frac{\chi}{2}\left[\left(q_{n+1}-q_{n}\right)+\left(q_{n}-q_{n-1}\right)\right],
$$

with $\chi=\chi_{0} / B$ as a new constant. This linear "ansatz" considers only the shifts due to the nearest lattice deformations.

In order to connect this approach with the polarization potential considered in the previous section we assume that the energy levels are shifted like the energies created by the polarization potential acting on the $n$-th electron from the side of the neighboring atoms. In order to compare this approach with the linear shifts used in the literature, we also consider the linear (small polaron) approximations to equation (9). The polarization approach gives in linear form

$$
\delta E_{n} \simeq U_{\mathrm{e}} \frac{64 h^{4} \sigma}{B\left(\sigma^{2}+4 h^{2}\right)^{3}}\left[\left(q_{n+1}-q_{n}\right)+\left(q_{n}-q_{n-1}\right)\right] .
$$

Comparing (14) and (15) we find

$$
\chi=U_{\mathrm{e}} \frac{128 \sigma h^{4}}{B\left(\sigma^{2}+4 h^{2}\right)^{3}} .
$$

Recall that $\chi_{0}=B \chi$. Then, for $U_{\mathrm{e}}=0.01 D, h=0.3 \sigma$, in the framework of the polarization model $\chi_{0} \simeq 0.003 D$. Assuming $D=1 \mathrm{eV}$, and $\sigma=2 \AA$ we obtain $\chi_{0}=0.002-0.003 \mathrm{eV} / \AA$. These calculations correspond to the parameter values: $\chi_{0}=0.005 D, h=0.3, U_{\mathrm{e}}=0.01$. As the parameter values in this approach are about one or two orders of magnitude below the earlier indicated values we expect that here polaron effects are rather weak and hence the system dynamics is dominated by solitons.

The probability to find the electron at the lattice site or atom located at $x_{n}$, i. e., the occupation number $p_{n}$, is $p_{n}=c_{n} c_{n}^{*}$. From the Hamiltonian follows the Schrödinger equation for the components of the wave function $c_{n}$ :

$$
\begin{aligned}
\mathrm{i} \frac{\mathrm{d} c_{n}}{\mathrm{~d} t}=\sum E_{n, m} c_{m}= & {\left[\epsilon_{0}+\delta \epsilon_{n}\left(q_{n+1}, q_{n-1}\right)\right] c_{n}-\tau\left\{\exp \left[-\alpha\left(q_{n+1}-q_{n}\right)\right] c_{n+1}\right.} \\
& \left.+\exp \left[-\alpha\left(q_{n}-q_{n-1}\right)\right] c_{n-1}\right\}
\end{aligned}
$$

where $\epsilon_{0}$ and $\delta \epsilon_{n}$ are now considered as dimensionless (units: $\hbar \omega_{0}$ ). The following dimensionless ratio of characteristic times was introduced

$$
\tau=\frac{V_{0}}{\hbar \omega_{0}}
$$


where $\omega_{0}$ is the classical oscillation frequency around the minima of the Morse potential. The corresponding Newtonian equations for the lattice units are

$$
\begin{aligned}
\frac{\mathrm{d}^{2} q_{n}}{\mathrm{~d} t^{2}}= & -p_{n} \frac{\partial \delta \epsilon_{n}\left(q_{n+1}, q_{n-1}\right)}{\partial q_{n}}+\left\{1-\exp \left[-\left(q_{n+1}-q_{n}\right)\right]\right\} \exp \left[-\left(q_{n+1}-q_{n}\right)\right] \\
& -\left\{1-\exp \left[-\left(q_{n}-q_{n-1}\right)\right]\right\} \exp \left[-\left(q_{n}-q_{n-1}\right)\right] \\
& -\alpha V_{0}\left\{\exp \left[-\alpha\left(q_{n}-q_{n-1}\right)\right]\left(c_{n+1}^{+} c_{n}+c_{n+1} c_{n}^{+}\right)\right. \\
& \left.+\exp \left[-\alpha\left(q_{n+1}-q_{n}\right)\right]\left(c_{n}^{+} c_{n-1}+c_{n} c_{n-1}^{+}\right)\right\} .
\end{aligned}
$$

The dynamical problem reduces, in principle, to solving both equations (17) and (19) coupled together. Examples of numerical solutions are shown in figure 5. Possible problems of consistency between classical and quantum dynamics remain, however, unsolved within this approach. Let us
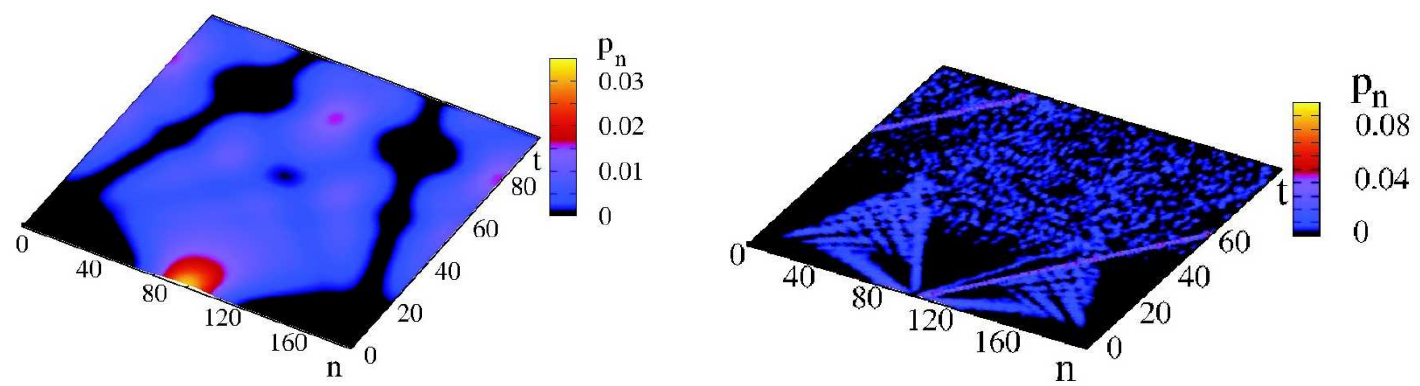

Figure 5. Morse lattice: Electron and solectron dynamics. Results of numerical integration of equations (17) and (19). Left panel: $\alpha=0$, dynamics of an electron without coupling to the lattice. Right panel: $\alpha=1,75$, dynamics of a solectron (an electron bound to the soliton).

now discuss solectronic excitations. We consider only one of the possible soliton-mediated processes: single electron transfer (ET) in a soliton-mediated hopping process along the lattice from a donor to an acceptor. When an added, excess electron is placed at a donor located at site $n=100$ at time $t=0$, figure 5 shows our findings. Due to the coupling, the electron is dynamically bound to the soliton which is the solectron excitation. When the electron-lattice interaction is operating, we see that the electron moves with the soliton with a slightly supersonic velocity $v_{\text {el }} \sim \frac{100}{70} v_{\text {sound }}$ and is running to the right border of the square plot. Let us assume that an acceptor is located there. This means that the electron is guided by the soliton from donor to acceptor. In reality, the electron cannot ride on just a single soliton from donor to acceptor. Several solitons should be involved in transport. We have already mentioned this kind of promiscuity of the electron. Therefore, the above given soliton velocity is an upper bound for the ET process. In principle, this effect may be used as a way of manipulating the transfer of electrons between donor and acceptor [31]. Here we offer a generalization of the phonon-assisted hopping process studied earlier by several authors [32-34]. Clearly, in our case we may have a polaron effect due to the electron-phonon (or soliton) interaction in addition to the genuinely lattice soliton effect due to the anharmonicity of the lattice vibrations. So far our method was based on a simultaneous solution of the classical equations for the Morse particles and the TBA-equations for the coefficients $c_{n}$ of the electronic wave function. As known, this procedure may lead to difficulties due to the lack of consistency between classical and quantum-mechanical dynamics [29,30]. Cruzeiro et al. [30] circumvented these problems by numerically solving the eigenvalue problem using the LAPACK procedure and going after each numerical step to the lowest eigenstate of the quantum problem. This corresponds to zero temperature, and we are interested here in finite temperatures. 


\section{Solectronic excitations in TBA}

\subsection{The eigenvalue problem of the TBA}

The TBA-matrix $E_{n, m}$ is of a special type. The matrix is quadratic and has elements only along the diagonal and near the diagonal. Such matrices are called band matrices with bandwidth three. Sometimes one speaks about triagonal matrices or matrices of Hessenberg type (see e. g. [35]. For our further studies we need the eigenvalues $\epsilon_{n}$ given by the solution of the determinant problem

$$
\left|E_{n m}-\lambda \delta_{n m}\right|=0
$$

The $N$ solutions for $\lambda$ we call the eigenvalues $\epsilon_{1}, \epsilon_{2}, \ldots, \epsilon_{N}$. The determinant of a tridiagonal matrix is known as a continuant [35]. In particular, the unperturbed case with constant elements $\epsilon_{0}$ in the diagonal and constant terms $\tau, q_{k}=0$ is solvable and gives a mobility band with the lower edge $\epsilon_{0}-2 \tau$. We are interested here in the perturbations due to the dynamics of the lattice. Then, all matrix elements depend on the elongations $q_{n}(t)$, the eigenvalues are the quickly changing functions of time $\epsilon_{n}(t)$. For definiteness we will assume the linear approximation for the diagonal elements

$$
\delta E_{n n}=\frac{\chi}{2}\left[\left(q_{n+1}-q_{n-1}\right)\right]
$$

and for the offdiagonal elements

$$
\delta E_{n, n+1}=\tau\left(1-\exp \left[-\alpha\left(q_{n+1}-q_{n}\right)\right]\right), \quad \delta E_{n, n-1}=\tau\left(1-\exp \left[-\alpha\left(q_{n}-q_{n-1}\right)\right]\right) .
$$

Solutions of the eigenvalue problem for special soliton-like excitations of $q_{n}(t)$ were found by Hennig et al. [24,25]. A numerical solution may be found with standard programs, e. g., LAPACK (see [35]). Since this is a difficult task [30], we also discuss approximate solutions of the eigenvalue problem. All approximations are based on the assumption that the off-diagonal elements representing the transitions are small in comparison with the diagonal elements. In this context the Gershgorin circle theorem is helpful. This theorem may be used to bound the spectrum of a square matrix [35]. One possible interpretation of this theorem is that if the off-diagonal entries of the square matrix are small (have small norms), the eigenvalues of the matrix cannot be "far from" the diagonal entries of the matrix. Let us assume that we have at time $t$ an excitation localized at the position $n$ of a long chain. Using the key idea of Gershgorin we assume that besides the diagonal elements $E_{0}$ only the elements in a small circle around the element $n-n$ are substantially perturbed due to elongations. We consider the matrix

$\begin{array}{ccccc}0 & 0 & 0 & 0 & 0 \\ 0 & 0 & \delta E_{n-1, n} & 0 & 0 \\ 0 & \delta E_{n, n-1} & \delta E_{n, n} & \delta E_{n, n+1} & 0 \\ 0 & 0 & \delta E_{n+1, n} & 0 & 0 \\ 0 & 0 & 0 & 0 & 0\end{array}$.

To find the eigenvalues of this matrix is a solvable problem which leads besides zeroes to two non-zero eigenvalues

$$
\delta \epsilon_{n}=\frac{1}{2} \delta E_{n} \pm \frac{1}{2}\left[\left(\delta E_{n}\right)^{2}+4 \delta E_{n, n+1} \delta E_{n+1, n}+4 \delta E_{n-1, n} \delta E_{n, n-1}\right]^{1 / 2}
$$

The lower one of these two eigenvalues is of physical interest. and we finally obtain

$$
\begin{aligned}
& \delta \epsilon_{n}\left(q_{n-1}, q_{n}, q_{n+1}\right)=\frac{\chi_{1}}{4}\left[q_{n+1}-q_{n-1}\right] \\
& \quad-\frac{1}{4}\left(\left[\chi_{1}\left(q_{n+1}-q_{n-1}\right)\right]^{2}+8 \tau^{2}\left[\exp \left[-2 \alpha\left(q_{n+1}-q_{n}\right)\right]+\exp \left[-2 \alpha\left(q_{n}-q_{n-1}\right)-2\right]\right]\right)^{1 / 2} .
\end{aligned}
$$

This way we have found an approximation which expresses the eigenvalue corresponding to the site $\mathrm{n}$ by the deviations of the nearest neighbours, which form a kind of Gershgorin circle with radius one. 


\subsection{Adiabatic canonical distributions in TBA}

In the first approximation with non-interacting electrons the canonical equilibrium distribution is

$$
p_{n}^{0}=\exp \left[\beta\left(\mu-\epsilon_{n}\right)\right],
$$

where $\epsilon_{n}$ are the eigenvalues at a given time. The chemical potential $\mu$ is given by the normalization. In the adiabatic approximation we assume that this distribution is reached in a very short time. Using the approximation (21) we get

$$
p_{n}^{0} \simeq \exp \left[-\frac{\delta E_{n}}{k_{\mathrm{B}} T}\right]=\exp \left[-\frac{\hbar \omega_{0} \chi\left(q_{n+1}-q_{n-1}\right)}{k_{\mathrm{B}} T}\right] .
$$

This zeroth approximation gives only a qualitative estimate, while a better first approximation is given by equation (24). Suppose that one big soliton is excited and assume the following shape of the solution for Morse solitons (with $b_{\text {eff }}=3 b$ ) [19]

$$
\exp \left[-3\left(q_{n}-q_{n-1}\right)\right]=1+\beta_{0}^{2} \cosh ^{-2}\left[\kappa n-\beta_{0} t\right]
$$

By introducing this into the zeroth approximation (26) we find

$$
p_{n}^{0} \simeq\left[1+\beta_{0} \cosh ^{-2}\left[\kappa n-\beta_{0} t\right]\right]^{\zeta}\left[1+\beta_{0} \cosh ^{-2}\left[\kappa(n+1)-\beta_{0} t\right]\right]^{\zeta},
$$

where

$$
\zeta=\frac{\chi}{6 k_{\mathrm{B}} T}
$$

We see that a thermally excited soliton is quite similar to a normal mechanically excited soliton except for some kind of a twin structure and a little deformation of the shape and the amplitude, both temperature-dependent. The velocity of such thermal soliton is the same as the standard soliton velocity.
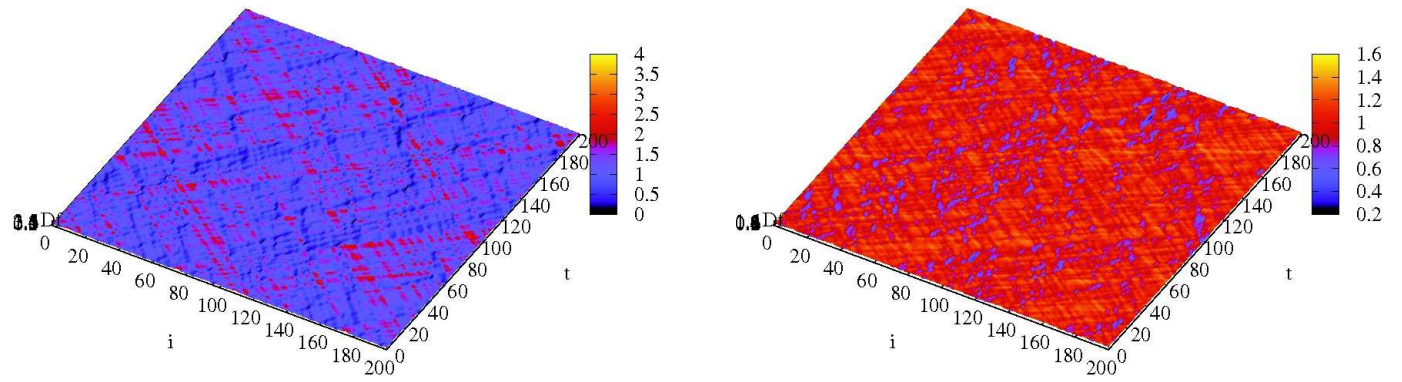

Figure 6. Toda-Morse lattice. Probability distribution of an electron in a heated anharmonic lattice in the adiabatic approximation according to the quantum canonical distribution. The actual time evolution of the distribution is displayed. Left figure: $T=0.1$; right figure: $T=0.5$. Parameter values: $B \sigma=1, \alpha=1.75, V_{0}=1, \tau=10$ and $\gamma=0.002$

In thermal systems. the canonical equilibrium distribution in the adiabatic approximation is given by the time-dependent energy eigenvalues. Introducing here the approximation based on Gershgorin circles of radius 1 we get a rather complicated but fully analytical formula for the time-dependent occupations

$$
\begin{aligned}
p_{n}(t) & \simeq \exp \left[-\frac{\hbar \omega_{0} \chi}{4 k_{\mathrm{B}} T}\left[q_{n+1}-q_{n-1}\right]+\frac{\hbar \omega_{0}}{4 k_{\mathrm{B}} T}\right. \\
& \left.\times\left(\left[\chi\left(q_{n+1}-q_{n-1}\right)\right]^{2}+8 \tau\left[\exp \left[-2 \alpha\left(q_{n+1}-q_{n}\right)\right]+\exp \left[-2 \alpha\left(q_{n}-q_{n-1}\right)-2\right]\right]\right)^{1 / 2}\right] .
\end{aligned}
$$

Here the displacements have to be taken from computer simulations of thermal solitonic excitations. The distribution is a quickly changing local function of the displacements. In the adiabatic 
approximation we assume that this distribution is reached in a very short time. An example is shown in figure 6 . Noteworthy is that this picture of a canonical quantum distribution is qualitatively quite similar to the classical distributions shown in an earlier section. We may estimate the soliton frequency from the thermal statistics of the solitons [7,8] for Toda interactions: (i) single solitons with parameter $\kappa$ are described by equation (27); and (ii) the density of solitons depending on parameter $\kappa$ is known.

\section{Discussion and concluding remarks}

We have demonstrated here for simple one- and two-dimensional molecular systems with exponential repulsive interactions that an interplay between electron motion and soliton excitations exists which may lead to special electron capturing effects and to relatively long correlations. We have shown the occurrence of a temperature region (near a characteristic lattice transition temperature $T_{\mathrm{tr}}$ which is around $10^{2} \mathrm{~K}$ for typical nonlinear $1 \mathrm{~d}$-lattices and for corresponding $2 \mathrm{~d}$-systems) where relatively numerous solitons are excited, and the strongest effects of coupling of electrons to the compressional regions are observed. Under such conditions most of the electron density is rather concentrated in clusters near the solitonic compressions. The electrons are attracted to the local compressions which justifies the concept of solectrons. In 1d-systems the clusters are always disconnected. Electricity may be carried only by the moving clusters and we cannot expect a 1d-system to go to highly conducting soliton-mediated states. However, we may expect the situation in $2 \mathrm{~d}-$ or quasi-2d systems to be quite different. As already mentioned, Astakhova and Vinogradov have derived a continuous description of these excitations by a suitable generalization of the KadomtsevPetviashvili equation [13]. What matters here is that the excitations in $1 \mathrm{~d}$ - and 2 d-systems are similar with respect to the local density enhancement and the corresponding local potential wells. An example of density droplets in a 2 d-system (obtained with the method of overlapping cores) is demonstrated in figure 7. So far we could not find the effects of percolation of the electronic density. However, with increasing density of the solitonic droplets, percolation should be possible, in principle. This hypothetical percolation effect would open the door to new interesting applications of transport effects which are stable to rather high temperatures lasting for several picoseconds.

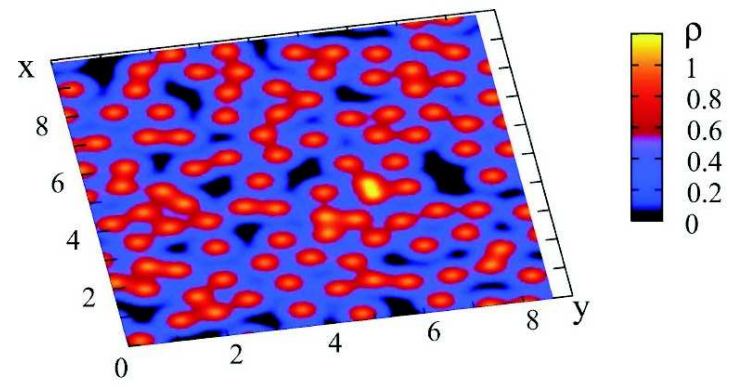

Figure 7. Two-dimensional system of Morse molecules. Example of an electron distribution in a heated anharmonic lattice $(T=0.2)$ with several locally percolating regions of high electron density. Parameters as in figure 3.

\section{Acknowledgements}

This research has been sponsored by the EU under Grant SPARK II-FP7-ICT-216227 and by the Spanish Government under Grant MEC-VEVES-FIS2006-01305. We thank L. Brizhik, L. Cruzeiro-Hansson, R. Feistel, D. Hennig, and G.A. Vinogradov for helpful discussions 


\section{References}

1. Bogolyubov N.N. Problems of dynamical theory in statistical physics. OGIS, Mosow, 1946 (in Russian); Selected Works, vol. 1-3. Naukova Dumka, Kyiv, 1969-1970 (in Russian).

2. Davydov A.S. Solitons in Molecular Systems, 2nd edn. Reidel, Dordrecht, 1991.

3. Christiansen A.L., Scott A.C. (eds.) Davydov's Soliton Revisited. Self-trapping of Vibrational Energy in Protein. Plenum Press, New York, 1983.

4. Lomdahl P.S., Kerr W.C., Phys. Rev. Lett., 1985, 55, 1235.

5. Scott A.C., Phys. Rep., 1992, 217, 1-67.

6. Toda M. Nonlinear Waves and Solitons. KTK Scientific Publishers, Tokyo, 1989; Theory of Nonlinear Lattices, 2nd edn. Springer-Verlag, New York, 1989.

7. Mertens F.G., Büttner H. - In: Solitons, edited by S.E. Trullinger, V.E. Zakharov and V.L. Pokrovsky. North-Holland, Amsterdam, 1986, ch. 15.

8. Marchesoni F., Lucheroni C., Phys. Rev. B, 1991, 44, 5303.

9. Zolotaryuk A.V., Spatschek K.H., Savin A.V., Phys. Rev. B, 1996, 54, 266.

10. Ebeling W., Chetverikov A., Jenssen M., Ukrain. J. Phys., 2000, 45, 479.

11. Nepomnyashy A.A., Velarde M.G., Phys. Fluids, 1994, 6, 187.

12. Nekorkin V.I., Velarde M.G. Synergetic Phenomena in Active Lattices, Patterns, Waves, Solitons, Chaos. Springer Verlag, Berlin 2002.

13. Astakhova T.Yu., Vinogradov G.A., J. Phys. A Math. Gen., 2006, 39, 3593.

14. Zhen W., Hongqing Z., Chaos, Solitons \& Fractals, 2007, 31, 197.

15. Fugmann S., Hennig D., Schimansky-Geier L., Hänggi P., Phys. Rev. E, 2008, 77, 061135.

16. Ebeling W., Podlipchuk V., Z. Physik. Chem., 1996, 193, 207.

17. Ebeling W., Valuev A.A., Podlipchuk V.J., J. Molec. Liquids, 1997, 74, 445.

18. Ebeling W., Sapeshinsky M., Valuev A., Int. J. Bifurc. \& Chaos, 1998, 8, 755.

19. Chetverikov A.P., Ebeling W., Velarde M.G., Eur. Phys. J. B, 2005, 44, 509;ibid., 2006, 51, 87.

20. Velarde M.G., Ebeling W., Chetverikov A.P., Int. J. Bifurcation Chaos, 2005, 15, 245.

21. Chetverikov A.P., Ebeling W., Velarde M.G., Int. J. Bifurcation Chaos, 2006, 16, 1613.

22. Chetverikov A.P., Ebeling W., Röpke G., Velarde M.G., Contr. Plasma Phys., 2007, 47, 465.

23. Velarde M.G., Ebeling W., Hennig D., Neissner C., Int. J. Bifurcation Chaos, 2006, 16, 1035.

24. Hennig D., Neissner C., Velarde M.G., Ebeling W., Phys. Rev. B, 2006, 73, 024306.

25. Hennig D., Chetverikov A., Velarde M.G., Ebeling W., Phys. Rev. E, 2007, 76, 046602.

26. Velarde M.G., Ebeling W., Chetverikov A.P., Hennig D., Int. J. Bifurcation Chaos, 2008, 18, 521.

27. Velarde M.G., Neissner C., Int. J. Bifurcation Chaos, 2008, 18, 885.

28. Heeger A.J., Kivelson S., Schrieffer J.R., Su W.P., Rev. Mod. Phys., 1988, 60, 781.

29. Cruzeiro-Hansson L., Phys. Rev. Lett., 1994, 73, 2927.

30. Cruzeiro-Hansson L., Takeno S., Phys. Rev. E, 1997, 56, 894.

31. Gray H.B., Winkler J.R., Proc. Natl. Acad. Sci. USA, 2005, 102, 3534.

32. Wan C., Fiebig T. et al., Proc. Natl. Acad. Sci. USA, 1999, 96, 6014;ibid., 2000, 97, 14052.

33. Kalosakas G., Aubry S., Tsironis G.P., Phys. Rev. B, 1998, 58, 3094.

34. Kalosakas G., Rasmussen K.O., Bishop A.R., J. Chem. Phys., 2003, 118, 3731; Synthetic Metals, 2004, $141,93$.

35. Horn R.A., Johnson C.R. Matrix analysis. Cambridge University Press, Cambridge, 1985.

36. Chetverikov A.P., Ebeling W., Velarde M.G., Eur. Phys. J. B, 2009, 70, 217. 


\title{
Зв'язані стани електронів і збуджень солітонного типу у теплових системах: адіабатичні наближення
}

\author{
В. Ебелінгํㅗ. М.Г. Веларде ${ }^{2}$ А.П. Четверіков ${ }^{3}$
}

1 Інститут фізики, університет Гумбольдта, Берлін, Німеччина

2 Полідисциплінарний інститут, Мадридський університет Комплутенсе

3 Інститут фізики, Саратовський держаний університет, Саратов

Отримано 23 червня 2009 р.

Ми вивчаємо зв'язані стани електронів із солітонними збудженнями в одно- та двовимірних системах. Розглядаються взаємодії між атомами типу Морзе у температурному інтервалі від низьких до фізіологічних температур. Атоми розглядаються на основі класичних рівнянь Ланжевета. У першому підході місця стиску візуалізуються шляхом виявлення області перекриття густин основних електронів. Далі на основі аналізу руху вільних електронів щодо атомів досліджується ефект нелінійних коливань. У наближенні сильного зв'язку вивчено зв'язану класичну і квантово-механічну динаміку електронів на ґратці. Крім того, базуючись на аналізі спектру енергій та канонічного розподілу, розглянуто формування солелектронів, тобто динаміку зв'язаних електрон-солітонних станів в адіабатичних наближеннях.

Ключові слова: нелінійна ґратка, електрон-ґраткові взаємодії, солелектрони, адіабатичні наближення

PACS: $63.20 . R y, 05.60 .-k, 05.45 . Y v, 71.38 .-k$ 
